\title{
Molecular emission in the active galactic nucleus of NGC 1068
}

\author{
Emmanuel Galliano ${ }^{1}$ and Danielle Alloin ${ }^{1}$ \\ ${ }^{1}$ ESO, Casilla 19001, Santiago, Chile
}

\begin{abstract}
We provide an overview of a high spatial resolution study of the molecular emission $\left(\mathrm{H}_{2}\right.$ and $\left.\mathrm{CO}\right)$ in the few central arcsec region around the AGN in NGC1068. A 0.5" resolution $\mathrm{H}_{2} 2.12 \mu \mathrm{m}$ line map obtained with VLT/ISAAC is presented and reveals interesting characteristics: two unequally bright knots located at $1^{\prime \prime}(70 \mathrm{pc})$ from the nucleus, along a direction perpendicular to the ionizing cone axis. We propose a model involving differential extinction, produced in the emitting molecular disc itself, to explain the intensity difference between the two knots. This effect can arise from a simple configuration, in which the orientation of the molecular disc is slightly tilted with respect to that of the central Compton thick absorber, which blocks the X-rays along our line of sight.
\end{abstract}

\section{Introduction}

High spatial resolution NIR instruments allow us to study the morphology of the warm molecular emission, through $\mathrm{H}_{2}$ line emission, in the environment of nearby AGNs. This complements CO emission line studies, which, thanks to millimeter interferometers, like the IRAM array, can produce $0.5^{\prime \prime}$ resolution maps. The morphologies of continuum emission on one hand, and molecular and atomic line emission on the other hand, must be used to constrain AGN models. New instruments (NACO, VLTI, SINFONI) are opening doors towards a promising high resolution view of the dusty and molecular environment of nearby AGNs.

Using VLT/ISAAC, we have obtained a high resolution map of the $\mathrm{H}_{2}$ emission around the nucleus of NGC1068, and interpret it with a simple excitation model allowing to reproduce the complex morphology of the observed maps. NGC1068 is a nearby type 2 AGN, where the projected angular scale is $70 \mathrm{pc}$ per arcsec.

\section{Mapping the $\mathbf{H}_{2}$ emission}

The $\mathrm{H}_{2}$ emission in NGC1068 was mapped with ISAAC on the VLT, at high angular and spectral resolution $\left(0.5^{\prime \prime}\right.$ and $35 \mathrm{~km} \mathrm{~s}^{-1}$, respectively). Long slit spectra were obtained over the whole central $4^{\prime \prime} \times 4^{\prime \prime}$ region at two perpendicular position angles. Details about the observing technique and results are published in Alloin et al. (2001) and Galliano \& Alloin (2002).

The map of the $\mathrm{H}_{2}$ emission is shown in Fig. 1. The emission is distributed mainly along a direction perpendicular to the ionizing cone (N-S direction) and to the extended MIR emission (Alloin et al. 2000). Two bright $\mathrm{H}_{2}$ knots are found at $\sim 1^{\prime \prime}$ (70 pc) from the nucleus, along the E-W direction. The sources are located symmetrically around the K-band continuum peak, which coincides with the AGN central engine, where no $\mathrm{H}_{2}$ emission is detected. The profiles of the $\mathrm{H}_{2}$ emission line arising from the two knots are symmetric in velocity, and show a difference of $140 \mathrm{~km} \mathrm{~s}^{-1}$ between their peaks, which can be interpreted as rotation of the molecular material around the axis of the ionization 


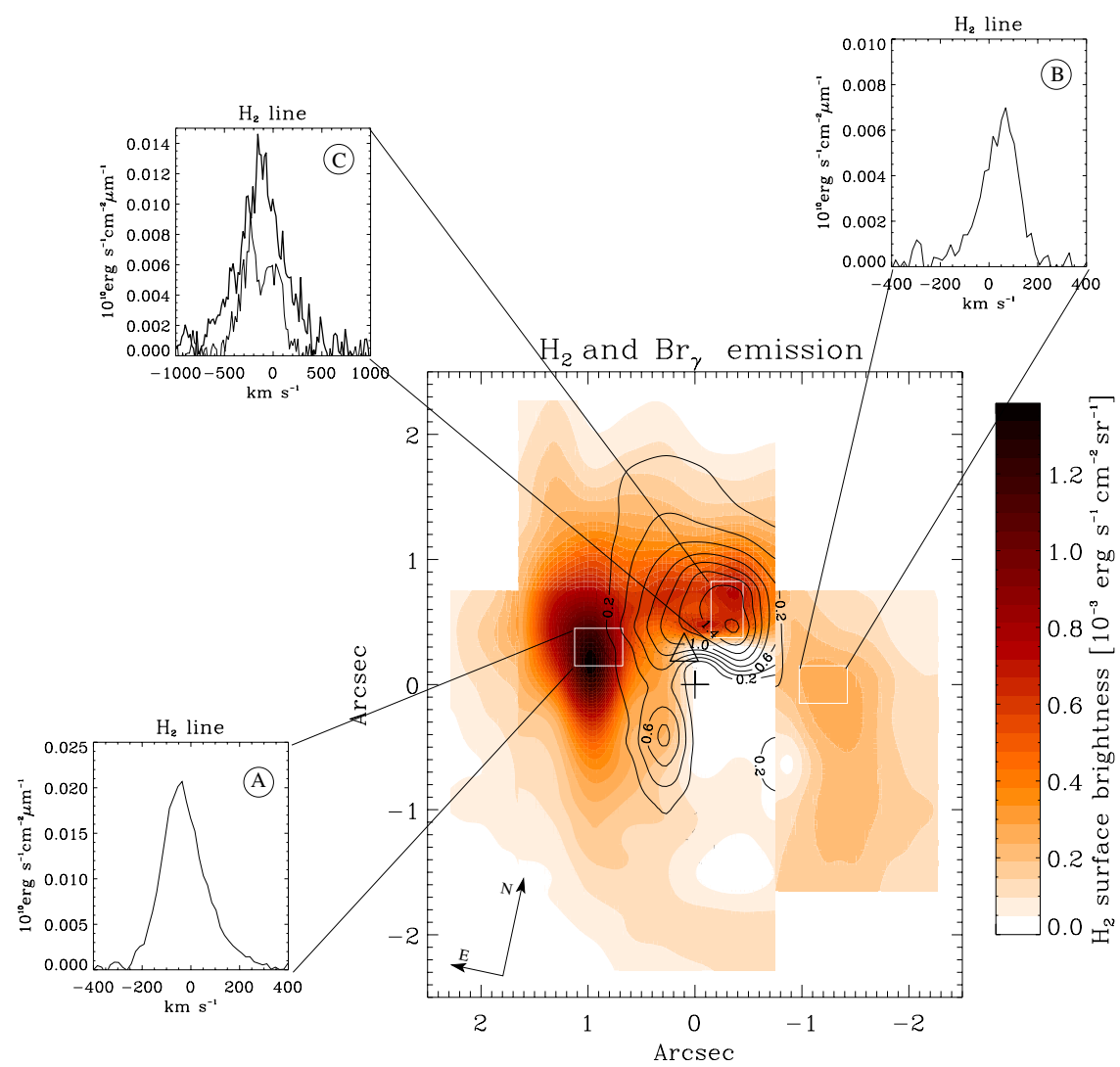

Figure 1. The central region of NGC1068. $\mathrm{H}_{2}$ emission: red image and thin profiles; $\mathrm{Br} \gamma$ emission: contour image and thick profiles. The central cross marks the position of the central engine.

cone. The symmetry between the two knots does not hold anymore when looking at the line intensity: the eastern knot is three times brighter than the western knot.

The simplest explanation for this intensity difference would be to assume a difference in the amount of molecular material between the two knots. However, the CO map obtained by Schinnerer et al. (2000) with the IRAM interferometer shows a very similar morphology, except that in the CO line the intensity difference is smaller than the one observed in the $\mathrm{H}_{2}$ line: the East/West ratio is 3 for $\mathrm{H}_{2}$, while for $\mathrm{CO}$ it is only 1.5. This suggests that differential extinction also plays a role.

\section{Modeling the molecular line emission characteristics}

We show in this section that a pronounced differential extinction effect can arise from a simple configuration. For more details, see Galliano et al. (2003).

In NGC1068, and probably in most AGNs, the excitation of the molecular emission lines is dominated by X-rays from the central engine. Maloney et al. (1996) computed the physical conditions in a molecular cloud irradiated by X-rays. Using their results, we have modeled a disc of molecular clouds and predicted molecular line maps for different configurations, carefully taking into account self attenuation of the line photons by the disc itself. The other component in the model is the Compton thick absorber located in 


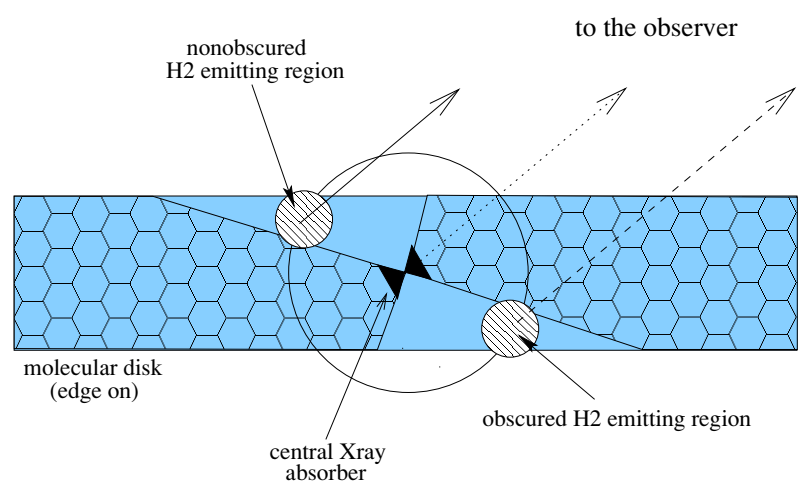

Figure 2. Model configuration sketch (see text).

the very central region of the AGN, and responsible for blocking the X-ray photons in our direction (Matt et al. 1997).

The model we propose is sketched in Fig. 2: (1) a large molecular disc, the edge of which is represented by the grey rectangle, and (2) a central Compton thick absorber, featured by the black component with conical shape. Due to the presence of the X-ray absorber, the regions marked with hexagons do not directly see the hard radiation from the central engine. The white circles show the locations where the excitation of the $\mathrm{H}_{2}$ molecules corresponds to the maximum of $\mathrm{H}_{2}$ line emission. The line photons produced in the disc region, on the right side of the sketch, have to cross the disc before reaching the observer, and hence are partly absorbed. The NIR $\mathrm{H}_{2}$ photons can be absorbed both by dust and molecules in velocity coincidence, while the mm CO photons are only affected by the intervening molecules in velocity coincidence. The intensity difference between the two regions is hence smaller for $\mathrm{CO}$ than for $\mathrm{H}_{2}$. Fig. 3 shows the orientation of the two components (the discs outlined show the orientation of the components but not their size) and compares the observed $\mathrm{H}_{2}$ and $\mathrm{CO}$ maps to the predicted ones. This model allows us to reproduce very well the $\mathrm{H}_{2}$ morphology, while the predicted ratio for $\mathrm{CO}$ remains smaller than observed. This suggests that a difference in the amount of material might indeed be present, in addition to differential extinction.

\section{Perspectives}

Detailed modeling of the molecular/dusty environment of AGNs should now include predictions for the molecular line emission distribution. Since 2-D spectrometers like SINFONI on the VLT will soon allow to map, in the $1-2.5 \mu \mathrm{m}$ range, the distribution of line emission at $0.1^{\prime \prime}$ resolution, the number of constraints will greatly increase in comparison to spectral energy distribution analysis. We believe that this approach will be very powerful for unveiling the distribution of cold material in the environment of AGNs. This is a necessary step to understand to which extent differences between AGN types are due to orientation effects, and later undertake evolutionary studies.

\section{References}

Alloin, D., et al. 2001, A\&A, 369, L33

Alloin, D., Pantin, E., Lagage, P. O., \& Granato, G. L. 2000, A\&A, 363, 926

Galliano, E., \& Alloin, D. 2002, A\&A, 393, 43

Galliano, E., Alloin, D., Granato, G., \& Villar-Martín, M. 2003, A\&A, 412, 615 
molecular disk orientation

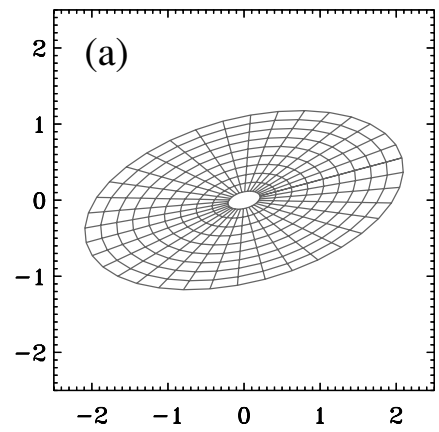

H2 intensity

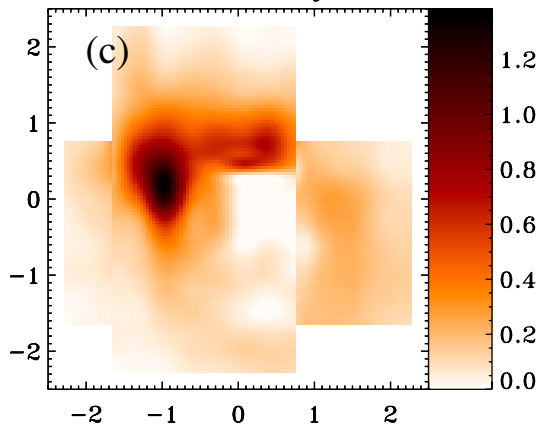

\section{CO intensity}

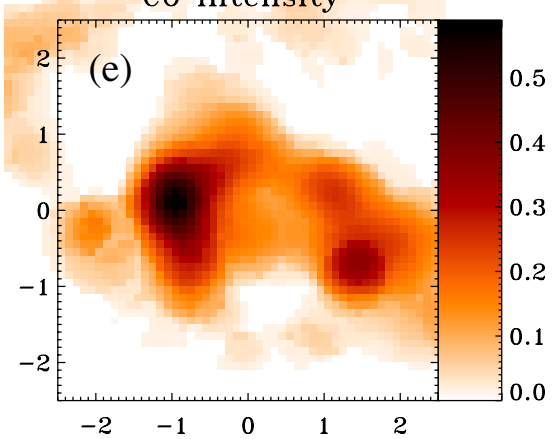

$\mathrm{X}$-ray absorber orientation

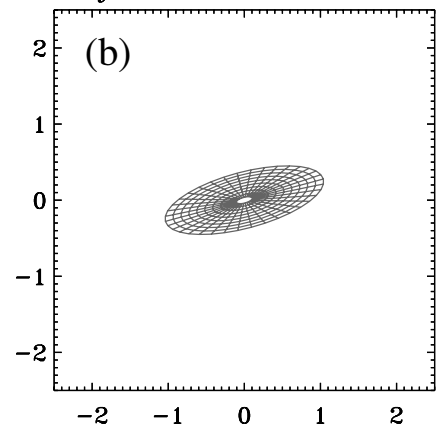

H2 intensity

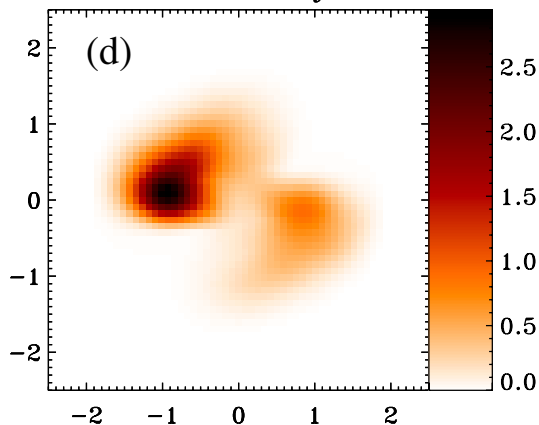

CO intensity

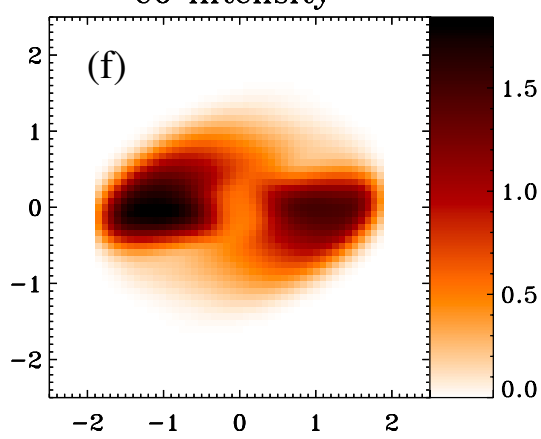

Figure 3. Observed maps compared to model maps. (a) orientation of the molecular disc; (b) orientation of the X-ray absorber (this component has no intrinsic size); (c) Observed $\mathrm{H}_{2}$ map; (d) Modeled $\mathrm{H}_{2}$ map; (e) Observed CO map; (f) Modeled CO map. The spatial scale is in arcsec, the brightness in $10^{-3} \mathrm{erg}^{-1} \mathrm{~cm}^{-2} \mathrm{sr}^{-1}$. North is up, East to the left. Reference for the CO data: Schinnerer et al. (2000)

Maloney, P. R., Hollenbach, D. J., \& Tielens, A. G. G. M. 1996, ApJ, 466, 561

Matt, G., et al. 1997, A\&A, 325, L13

Schinnerer, E., Eckart, A., Tacconi, L. J., Genzel, R., \& Downes, D. 2000, ApJ, 533, 850 